\title{
The Connection between the Narrow-Line Region and the UV Absorbers in Seyfert Galaxies ${ }^{1}$
}

\author{
D.M. Crenshaw \\ Department of Physics and Astronomy, Georgia State University, Atlanta, GA 30303; \\ crenshaw@chara.gsu.edu \\ S. B. Kraemer \\ Catholic University of America and Laboratory for Astronomy and Solar Physics, NASA's \\ Goddard Space Flight Center, Code 681, Greenbelt, MD 20771; \\ stiskraemer@yancey.gsfc.nasa.gov
}

\begin{abstract}
We present evidence that the outflowing UV absorbers in Seyfert 1 galaxies arise primarily in their inner narrow (emission) line regions (NLRs), based on similarities in their locations, kinematics, and physical conditions. 1) Hubble Space Telescope observations show that nearly all Seyfert galaxies have bright, central knots of [O III] emission in their NLRs with radii of tens of parsecs. These sizes are consistent with most previous estimates of the distances of UV (and X-ray) absorbers from their central continuum sources, and a recentlyobtained reliable distance of $\sim 25$ pc for a UV absorber in the Seyfert 1 galaxy NGC 3783. 2) The nuclear emission-line knots in a sample of 10 Seyfert galaxies have velocity widths of $300-1100 \mathrm{~km} \mathrm{~s}^{-1}$ (half-width at zero intensity), similar to the radial velocities of most UV absorbers. The highest radial velocity for a Seyfert UV absorber to date is only $-2100 \mathrm{~km} \mathrm{~s}^{-1}$, which is much lower than typical broad-line region (BLR) velocities. There is also mounting evidence that the NLR clouds are outflowing from the nucleus, like the UV absorbers. 3) If our hypothesis is correct, then the NLR should have a component with a high global covering factor $\left(C_{g}\right)$ of the continuum source and BLR, to match that found from previous surveys of UV absorbers $\left(C_{g}=0.5-1.0\right)$. Using STIS spectra of NGC 4151, obtained when the continuum and BLR fluxes were low, we find evidence for optically thin gas in its nuclear emission-line knot. We are able
\end{abstract}

\footnotetext{
${ }^{1}$ Based on observations made with the NASA/ESA Hubble Space Telescope, obtained at the Space Telescope Science Institute, which is operated by the Association of Universities for Research in Astronomy, Inc., under NASA contract NAS 5-26555.
} 
to match the line ratios from this gas with photoionization models that include a component with $C_{g} \approx 1$ and an ionization parameter and hydrogen column density that are typical of UV absorbers.

Subject headings: galaxies: Seyfert - ultraviolet:galaxies

\section{Introduction}

Mass outflows of ionized gas from active galactic nuclei (AGN) have been detected in the majority of Seyfert 1 galaxies observed in the UV (Crenshaw et al. 1999; Kriss 2002) and Xrays (Reynolds 1997, George et al. 1998). The outflows are revealed through absorption lines that are blueshifted with respect to the systemic velocities of the host galaxies. Observations at high spectral resolution $(\lambda / \Delta \lambda \gtrsim 10,000)$ in the UV show that the absorption tends to split into multiple kinematic components with widths in the range $20-500 \mathrm{~km} \mathrm{~s}^{-1}$. Detailed studies of the "intrinsic" absorbers indicate large global covering factors $\left(C_{g}=0.5-1.0\right)$ of the nucleus and mass-loss rates compable to the inferred mass accretion rates $(\sim 0.01$ $\mathrm{M}_{\odot} \mathrm{yr}^{-1}$ ) (Crenshaw et al. 2003a). The origin of the intrinsic absorbers (e.g., accretion disk, torus) and the means by which they are accelerated outward (e.g., thermal winds, radiatively-driven flows, hydromagnetic flows) are currently unknown.

The large global covering factors of the UV absorbers suggest that they should contribute to at least some of the UV and optical emission that we see. Thus, it is only natural to ask if they are associated in some way with either the narrow (emission) line region (NLR) or the broad (emission) line region (BLR). An important clue is that the highest radial velocity observed in any Seyfert 1 galaxy is only-2100 $\mathrm{km} \mathrm{s}^{-1}$ (Crenshaw et al. 1999), which is comparable to the maximum velocity (half-width at zero intensity) of the NLR gas and much less than that of the BLR gas, which extends out to $\gtrsim 10000 \mathrm{~km} \mathrm{~s}^{-1}$. Furthermore, the available evidence indicates that UV absorbers have transverse velocities comparable to their radial velocities (Crenshaw et al. 2003a, 2004a). Thus, the kinematics of the absorbers suggest that they are more closely related to the NLR than the BLR.

Another clue to the origin of the absorbers and their connection to the emission-line regions is their radial location with respect to the central supermassive black hole (SMBH), but this is a difficult parameter to obtain. Early studies of the ultraviolet spectrum of NGC 4151 with the International Ultraviolet Explorer found that the UV absorbers must lie outside of the broad (emission) line region (BLR), since both continuum and broad-line 
emission were absorbed (Bromage et al. 1985). This was later found to be the case as well for a sample of Seyfert 1 galaxies observed by the Hubble Space Telescope (HST) (Crenshaw et al. 1999). Various studies have placed upper or lower limits on the distances of outflowing UV or X-ray absorbers from their nuclei, and these fall in the range of tenths to tens of parsecs $^{1}$ (Kriss et al. 1997; Espey et al. 1998; Netzer et al. 2002; Kraemer et al. 2002; Gabel et al. 2003a; Netzer et al. 2003).

Recently, we determined a reliable distance for an outflowing UV absorber (Gabel et al. 2004a), which is Component "1" in NGC 3783, at a radial velocity of $-1350 \mathrm{~km} \mathrm{~s}^{-1}$ with respect to the systemic velocity of the host galaxy. From the relative strengths of the metastable C III* $\lambda 1175$ multiplet lines in absorption, we determined that the hydrogen number density of the absorber was $n_{H} \approx 4 \times 10^{4} \mathrm{~cm}^{-3}$. Combined with the ionization parameter and ionizing luminosity, this yields a distance of $\sim 25 \mathrm{pc}$ from the central continuum source (Gabel et al. 2004a). This absorber is therefore in the inner NLR, which typically extends out to several hundred parsecs from the nucleus (Schmitt et al. 2003a, b).

Given their locations and velocities, are the UV absorbers just NLR clouds seen in absorption? Global estimates of the covering factor of the NLR in Seyfert 1 galaxies yield values of $C_{g} \approx 0.02$ (Netzer \& Laor 1993). Furthermore, NLR clouds tend to have a lower ionization parameter $(U)$, defined as the ratio of ionizing photons to hydrogen atoms at the ionized face, and higher hydrogen column density $\left(N_{H}\right)$ than UV absorbers, on average (Crenshaw et al. 2003a). However, previous studies have already shown that a typical NLR is composed of a multi-phase medium; clouds with different physical conditions $\left(U, N_{H}, n_{H}\right)$ coexist at the same radial locations (Kraemer \& Crenshaw 2000, Kraemer et al. 2000b). One possibility is that UV absorbers are associated with a high-ionization, diffuse component of the NLR, rather than the entire NLR. This idea is similar to that of Cecil et al. (2002), who suggest that the high-velocity (extending up to $-3200 \mathrm{~km} \mathrm{~s}^{-1}$ ) emission-line clouds in the NLR of NGC 1068 would resemble the "associated absorbers" in quasars if seen against the continuum source. However, these high-velocity clouds have a covering factor of $C_{g} \leq 0.02$. In this paper, we investigate the possibility that there is a high-ionization component of the NLR with a covering factor that is sufficiently large to account for most of the intrinsic UV absorption in Seyfert galaxies.

HST images of the NLR show that nearly all Seyfert 1 and 2 galaxies have a bright central knot of [O III] emission (Schmitt et al. 2003a), and Space Telescope Imaging Spectrograph

\footnotetext{
${ }^{1} \mathrm{By}$ contrast, intrinsic absorption lines that are within a few hundred $\mathrm{km} \mathrm{s}^{-1}$ of the systemic velocity can often, but not always, be attributed to gas at much larger distances in the host galaxy's disk or halo (Weymann et al. 1997; Crenshaw \& Kraemer 2001).
} 
(STIS) slitless spectra of these nuclear knots show they have high velocity dispersions (Ruiz et al. 2005). Their high surface brightnesses, proximity to their nuclei, and high velocity dispersions indicate they are good candidates for UV absorbers seen in emission. In this paper, we explore a number of their properties based on the STIS spectra of Ruiz et al. (2005). To demonstrate that the nuclear emission-line knots can be responsible for the UV absorption, it is necessary to show that their spectra show evidence for a high-ionization, high covering factor component. We present a STIS UV/optical spectrum of the nuclear [O III] knot in NGC 4151 that is ideal for this purpose ( $\$ 4)$. First, however, we provide new measurements that explore the kinematic and positional similarities between the UV absorbers (§2) and the NLR (§3).

\section{Kinematics of the UV Absorbers}

Observations at high spectral resolution $(\lambda / \Delta \lambda \geq 10,000)$ are needed to resolve the kinematic components of intrinsic absorption in Seyfert galaxies. This requirement is met in the UV $(1200-3200 \AA)$ by observations with the high-resolution modes of HST's STIS or the first-generation Goddard High-Resolution Spectrograph (GHRS), and in the far-UV (900 - $1200 \AA)$ with the Far Ultraviolet Spectroscopic Explorer (FUSE). We have compiled the available measurements of the radial-velocity centroid $\left(v_{r}\right)$, relative to the host galaxy, and full-width at half-maximum (FWHM) of the absorption depth for individual absorption components observed by these instruments. The sample contains 61 absorption components in 10 Seyfert 1 galaxies: NGC 3516 (Crenshaw et al. 1999; Kraemer et al. 2002), NGC 3783 (Kraemer et al. 2001a; Gabel et al. 2003), NGC 5548 (Mathur et al. 1999; Crenshaw et al. 2003), Mrk 509 (Kriss et al. 2000; Kraemer et al. 2003), NGC 4051 (Collinge et al. 2001), NGC 4151 (Weymann et al. 1997; Kraemer et al. 2001b), Mrk 279 (Scott et al. 2004; J.R. Gabel, 2004, private communication), NGC 7469 (Kriss et al. 2003), Akn 564 (Crenshaw et al. 2002), and NGC 4395 (Crenshaw et al. 2004b).

Figure 1 plots the FWHM vs. $v_{r}$ for each intrinsic absorption component in our sample. Although the sample is rather sparse, there is no clear trend; components at both high and low $\left|v_{r}\right|$ show a large range in FWHM. The tendency for most low (within $\pm 250 \mathrm{~km} \mathrm{~s}^{-1}$ )

$v_{r}$ components to have low $\left(<100 \mathrm{~km} \mathrm{~s}^{-1}\right)$ FWHM reflects the finding that a large fraction arise in the Seyfert host galaxies, as has been demonstrated for specific components in NGC 4151 (Weymann et al. 1997), Akn 564 (Crenshaw et al. 2002), and NGC 4395 (Crenshaw et al. 2004b). Furthermore, a galactic origin may very well explain all of the cases of positive $v_{r}$. However, some of these low $v_{r}$ components are likely intrinsic to the AGN; for example, a component in NGC 3516 at $v_{r}=30 \mathrm{~km} \mathrm{~s}^{-1}$ has shown strong variability in its column 
density, indicating that it arises close to the nucleus (Kraemer et al. 2002). ${ }^{2}$

For our purposes, the importance of Figure 1 is that it shows the radial velocities of intrinsic UV absorbers are $\left|v_{r}\right| \leq 2100 \mathrm{~km} \mathrm{~s}^{-1}$ in Seyfert galaxies, and the majority of components (51 out of 61 ) have $\left|v_{r}\right|<1000 \mathrm{~km} \mathrm{~s}^{-1}$. In a survey of low-resolution UV spectra of Seyfert galaxies, we found a similar result: the highest (most negative) radial velocity was $-2150 \mathrm{~km} \mathrm{~s}^{-1}$, for I Zw 1 (Crenshaw et al. 1999). Transverse velocities of the absorbers are more difficult to obtain, but a few lower limits on the order of $\sim 1000 \mathrm{~km} \mathrm{~s}^{-1}$ have been determined by detecting the motion of absorbing clouds across the BLR (Crenshaw et al. 2003a, and references therein). The only reasonably accurate transverse velocity to date is for "Component 1" in NGC 3783, which lies in the range $v_{T}=540-1430 \mathrm{~km} \mathrm{~s}^{-1}$ (Crenshaw, Kraemer, \& Gabel 2004a). Many other absorption components have been monitored on a yearly basis and have not shown evidence for transverse motion, so the transverse velocities could be much lower for these components. Although more work needs to be done, the available evidence indicates that the space velocities of UV absorbers in Seyfert galaxies are much less than those of BLR clouds, and very similar to the velocities in the NLR.

\section{Kinematics and Sizes of the Nuclear Emission-Line Knots}

We require the high spatial resolution of $H S T$ to isolate the nuclear emission-line knots and measure their sizes, and the spectral resolution of STIS to accurately measure their kinematics. STIS medium-dispersion spectra of the bright [O III] $\lambda 5007$ emission line are ideal for this purpose. We use the STIS slitless spectra of NGC 4151 (Hutchings et al. 1998; Kaiser et al. 2000) and nine other Seyfert galaxies (Ruiz et al. 2005; see this paper for details on the observations and data reduction), obtained with the G430M grating and an open aperture. The spectra have a spatial resolution of $0^{\prime \prime} .1$ (full-width at half-maximum) (FWHM) and a velocity resolution of $33 \mathrm{~km} \mathrm{~s}^{-1}$ (FWHM) in the dispersion direction. Ruiz et al. (2005) found that nine of the ten Seyfert galaxies in their sample show bright, compact $\left(\mathrm{FWHM}<0^{\prime \prime} .5\right)[\mathrm{O}$ III] knots at their optical continuum peaks with apparently high velocity dispersions. The only exception is Mrk 3, which shows several bright knots with high velocity

\footnotetext{
${ }^{2}$ There are several other aspects of Figure 1 that deserve comment. The FWHMs have not been corrected for instrumental resolution ( $\sim 15 \mathrm{~km} \mathrm{~s}^{-1}$ for FUSE, $\sim 8 \mathrm{~km} \mathrm{~s}^{-1}$ for STIS), so that lines with the lowest FWHM $\left(20-30 \mathrm{~km} \mathrm{~s}^{-1}\right)$ are just barely resolved. Some of the absorption lines are likely saturated, so the FWHM may overestimate their intrinsic velocity spread. The four points with low FWHM at $\sim-2100$ $\mathrm{km} \mathrm{s}^{-1}$ are subcomponents of an absorption feature in NGC 7469 (Kriss et al. 2003). The point with very large FWHM $\left(940 \mathrm{~km} \mathrm{~s}^{-1}\right)$ represents the transient absorption component D' in NGC 4151 (Kraemer et al. 2001a).
} 
dispersions within $\sim 1^{\prime \prime}$ of its optical nucleus; we do not use Mrk 3 for this study.

For the 10 nuclear emission-line knots, we measured the half-width at half-maximum (HWHM) of the [O III] $\lambda 5007$ emission in the dispersion (radial velocity) and cross-dispersion (spatial) directions. We also measured the half-width at zero intensity (HWZI) in the dispersion direction. We use these parameters rather than the full widths to compare with the UV absorber kinematics, because they are more appropriate for simple geometries. For an expanding shell, for example, the HWHM of the intensity profile in the spatial direction can be compared to the average absorber distance, and the HWZI in the spectral direction can be compared to the maximum absorber radial velocities. However, we note that direct comparisons can only be made by assuming very specific geometric and kinematic models.

Table 1 shows measurements of the nuclear knots in our sample, which includes two Seyfert 1, one Seyfert 1.9, and seven Seyfert 2 galaxies at redshifts $<0.03$. Three of the nuclear knots are only marginally resolved, and we list their HWHM sizes (in arcsecs and parsecs) as upper limits. The HWHM's range from 7 to $67 \mathrm{pc}$, similar to estimates for the distances of UV absorbers from their continuum sources, as described in $\S 1$. The HWZI's range from 310 to $1060 \mathrm{~km} \mathrm{~s}^{-1}$, similar to the range of maximum absorber velocities found in most Seyfert galaxies, as described in $\S 2$.

\section{Physical Conditions in the Nuclear Emission-Line Knot of NGC 4151}

\subsection{Observations and Emission-Line Ratios}

To determine the physical conditions in a nuclear emission-line knot, we would like to have extensive UV and optical coverage to get as many emission-line diagnostics as possible. We also require the high spatial resolution of HST to isolate the knot. Of the sources in Table 1, only NGC 4151 has low-dispersion STIS spectra covering a large wavelength range (1150 - 10,270 A). We searched the Multimission Archives at the Space Telescope Science Institute (MAST) and found two sets of low-dispersion spectra of the nucleus: one obtained on 1998 January 8/February 10 (published in Nelson et al. 2000) and one obtained on 2000 May $24 / 28$ (previously unpublished). The latter set was obtained when the UV and BLR fluxes were in a much lower state (continuum flux at $1465 \AA=2.0( \pm 0.3) \times 10^{-14} \mathrm{ergs} \mathrm{s}^{-1} \mathrm{~cm}^{-2}$ $\left.\AA^{-1}\right)$ compared to that of the 1998 observations $\left(24.1( \pm 0.5) \times 10^{-14} \mathrm{ergs} \mathrm{s}^{-1} \mathrm{~cm}^{-2} \AA^{-1}\right)$, and is therefore ideal for deconvolving emission lines with both broad and narrow components. The only drawback in using NGC 4151 is that it has strong intrinsic absorption lines that contaminate the narrow emission-line spectrum in the UV. In particular, absorption due to the host galaxy and/or halo (components $\mathrm{F}$ and $\mathrm{F}^{\prime}$ in Weymann et al. 1997) severely absorb 
the narrow emission components of the UV resonance lines of $\mathrm{L} \alpha, \mathrm{N}$ V, O I, C II, Si IV, C IV, and Mg II, to the extent that their NLR contributions are not measurable even in STIS echelle spectra (see Kraemer et al. 2001b). However, when NGC 4151 is in a high state, the unusual (for Seyferts) Balmer absorption lines nearly disappear (Hutchings et al. 2002). Thus, we use the high-state slitless spectra of $\mathrm{H} \beta$ and $\mathrm{H} \alpha$ obtained on 1997 July 15 (previously published in Kaiser et al. 2000; Hutchings et al. 2002) to deconvolve their narrow and broad emission components.

In Table 2, we list the STIS observations that we retrieved from the MAST for this analysis. The first four observations are the low-dispersion, long-slit spectra, which have a resolving power of $\lambda / \Delta \lambda \approx 1000$, and the last two are the medium-dispersion, slitless spectra of $\mathrm{H} \beta$ (plus [O III] $\lambda \lambda 4959,5007$ ) and $\mathrm{H} \alpha$ (plus [N II] $\lambda \lambda 6548,6584$ ), with $\lambda / \Delta \lambda \approx 9000$. We reduced the STIS low- and medium-dispersion spectra using the IDL software developed at NASA's Goddard Space Flight Center for the Instrument Definition Team. We identified and removed cosmic-ray hits using multiple images and removed hot or warm pixels by interpolation in the dispersion direction. We used wavelength calibration exposures obtained after each science observation to correct the wavelength scale for zero-point shifts. For the G750L spectra, we used a contemporaneous continuum-lamp exposure in the flat-fielding process to remove the fringes at the long-wavelength end. We extracted the spectra from the flux-calibrated CCD images using a bin height of 11 pixels $\left(0^{\prime \prime} .55\right)$ in the cross-dispersion direction, which corresponds to the full-width at zero-intensity (FWZI) of the [O III] nuclear knot from the G430M slitless spectrum. For the flux-calibrated MAMA images, we used the same extraction height of $0^{\prime \prime} .55$, which corresponds to 22 pixels on the MAMA detector. We combined the low-dispersion spectra in their regions of overlap to obtain a single spectrum from $1150-10,270 \AA$.

In Figure 2, we show the full low-dispersion spectrum of the nuclear emission-line knot in NGC 4151. Narrow emission lines covering a broad range in ionization are present, from [O I] to [Fe XI]; the ionization potential needed to create the latter is $\mathrm{IP}_{c}=262 \mathrm{eV}$. We see no evidence for even higher ionization lines, like the $[\mathrm{S} \mathrm{XII}] \lambda 7611$ line $\left(\mathrm{IP}_{c}=505 \mathrm{eV}\right)$ seen in the STIS spectrum of the hot spot in NGC 1068 (Kraemer \& Crenshaw 2000a). The broad components of the permitted lines are clearly evident but still unusually weak for NGC 4151. Although UV resonance lines are detected in emission, their fluxes are severely affected by the intrinsic absorption lines, as discussed previously.

We measured the fluxes of isolated emission lines by direct integration above local continuum fits. For narrow lines that were blended with other broad or narrow emission, we used the [O III] $\lambda 5007$ profile as a template to deconvolve the blends (see Crenshaw \& Peterson 1986). We used the medium-dispersion spectra to deconvolve the blend of narrow 
and broad $\mathrm{H} \beta$ and the blend of narrow and broad $\mathrm{H} \alpha$ and the [N II] lines. As shown in Figure 3, the narrow component of $\mathrm{H} \beta$, which we use as our standard for line ratios, was easily separated from the broad component in this medium-dispersion spectrum. Lines that we deblended in the low-dispersion spectra include the broad and narrow components of the $\mathrm{H}$ and He permitted lines and the semi-forbidden lines in the UV, as well as the narrow [S II] $\lambda \lambda 6716,6731$ lines and $\mathrm{H} \gamma$ plus [O III] $\lambda 4363$ lines. We determined the reddening of the the narrow emission lines from the He II $\lambda 1640 / \lambda 4686$ ratio, the Galactic reddening curve of Savage \& Mathis (1979), and an intrinsic He II ratio of 7.2. We determined uncertainties in the dereddened ratios from the sum in quadrature of the errors from three sources: photon noise, different reasonable continuum placements, and reddening.

Table 3 gives the observed and dereddened narrow-line ratios, relative to $\mathrm{H} \beta$, as well as the uncertainties in these ratios. The reddening determined from the He II ratio is only $\mathrm{E}(\mathrm{B}-\mathrm{V})=0.02 \pm 0.04$. The reddening correction has a very small effect on our line ratios, as shown in Table 3, and uncertainties in the correction have a negligible effect on our results. The reddening that we have determined can be attributed entirely to reddening in our Galaxy, which is $\mathrm{E}(\mathrm{B}-\mathrm{V})=0.03$ (Schlegel et al. 1998). Thus, there is no evidence for dust in the inner NLR of NGC 4151, although at other locations in the NLR, the observed reddening of the emission lines ranges from $\mathrm{E}(\mathrm{B}-\mathrm{V})=0.01$ to 0.36 (Kraemer et al. 2001b).

\subsection{Photoionization Models}

To explore the physical conditions and covering factor of the nuclear knot in NGC 4151, we generated photoionization models using the code Cloudy (Ferland et al. 1998). We modeled the emission-line gas as single-zoned slabs, directly irradiated by the central source. We used the same spectral energy distribution (SED) of the ionizing continuum radiation as we used previously for NGC 4151 (Kraemer et al. 2001b), which consist of broken power-laws of the form $F_{\nu} \propto \nu^{\alpha}$, where the spectral index $\alpha=-1.0$ for $\mathrm{h} \nu<13.6 \mathrm{eV}, \alpha=-1.4$ over the range $13.6 \mathrm{eV} \leq \mathrm{h} \nu<1 \mathrm{keV}$, and $\alpha=-0.5$ above $1 \mathrm{keV}$, and the luminosity in ionizing

photons is $2 \times 10^{53} \mathrm{~s}^{-1}$. We assumed roughly solar elemental abundances (e.g. Grevesse \& Anders 1989) and that the gas was free of cosmic dust. The models are parameterized in terms of the ionization parameter $(U)$, the hydrogen number density $\left(n_{H}\right)$, and the hydrogen column density $\left(N_{H}\right)$.

The emission-lines in Table 3 span a huge range in ionization and critical density, and it is clear that multiple model components are required. Since the nuclear knot is resolved, we used the radius inside of which one-half of the knot's [O III] emission is contained as a starting point for the distances of the components. This radius is $0^{\prime \prime} .15$, which corresponds 
to a projected distance of 9.5 pc from the central continuum source.

The dereddened He II $\lambda 4686 / \mathrm{H} \beta$ ratio in Table 3 is relatively high for photoionized gas, which suggests either 1) a much harder continuum SED than we have used, or 2) a strong contribution from a high-ionization, matter-bound (optically thin to hydrogenionizing radiation) component. There is no evidence for a much harder SED and, in fact, we have used the above SED to successfully model the emission-line ratios from other parts of the NLR in NGC 4151 (Kraemer et al. 2000). Furthermore, any SED that possesses a realistic EUV bump would boost both the He II and [ $\mathrm{Ne} \mathrm{V}$ ] emission, but what is required is a high $[\mathrm{Ne} \mathrm{V}] / \mathrm{He}$ II ratio (Table 3 ). This can only be achieved with an optically thin component that has a large $\mathrm{Ne}^{+4}$ zone prior to the point where the gas becomes optically thick to the He II ionizing radiation (Kraemer \& Crenshaw 2000; Kraemer et al. 2000).

We have therefore adopted a matter-bound component ("UVABS") with parameters typical of most UV absorbers (Crenshaw et al. 2003): $U=0.03$ and $N_{H}=10^{19.5} \mathrm{~cm}^{-2}$ (with the above parameters, $n_{H}$ is fixed to $10^{4.3} \mathrm{~cm}^{-3}$ ). The predicted ionic column densities listed in the footnotes to Table 3 would result in absorption lines that are typical of most UV absorbers: strong-to-saturated Ly $\alpha$, C IV $\lambda \lambda$ 1548.2, 1550.8, N V $\lambda \lambda 1238.8,1242.8$, and O VI $\lambda \lambda 1031.9,1036.3$, and weak or undetectable Si IV $\lambda \lambda 1393.8,1402.8$.

In order to fit the observed emission-line ratios, we required two additional components: a moderate density, radiation-bound component ("OIII") to reproduce the strong [O III] $\lambda 5007$ emission, and a higher density, radiation-bound component ("DENSE") to account for the strong [O III] $\lambda 4363$ line (see Table 3). We were unable to find a satisfactory singlecomponent solution, since, for example, the [Ne IV] $\lambda 2423$ line is quenched at intermediate densities. The OIII component was kept at a distance of $9.5 \mathrm{pc}$, consistent with measurements of the [O III] $\lambda 5007$ emission. We kept the same ionization parameter $(U=0.01)$ for the DENSE component, but increased its density, which effectively moves it to a distance of 2.0 pc from the continuum source. To produce a composite model spectrum, we adjusted the weight of each component according to the fraction of $\mathrm{H} \beta$ it contributes until we obtained a good match with the dereddened line ratios. We give the component and composite line ratios in Table 3, along with the final model parameters, the weight of each component, and the flux of $\mathrm{H} \beta$ at the ionized face of each component $\left(f_{H \beta}\right)$.

Nearly all of the dereddened line ratios are fit well by the composite model. The low critical density lines (see Osterbrock 1989) from low ionization species such as [O II] $\lambda 3227$ and [S II] $\lambda \lambda 6716,6731$ are underpredicted, which could be rectified by introducing an additional component outside the emission-line bicone that is irradiated by a heavily absorbed continuum, similar to the model described for the hot spot in NGC 1068 (Kraemer \& Crenshaw 2000a). Also, the model significantly underpredicts the strengths of the [Fe VII] and 
[Fe XI] lines, but this is a common problem in NLR models (Oliva 1997) that may be due to problems with the atomic data (S.B. Kraemer \& G.J. Ferland, 2004, in preparation).

Table 3 shows that the inclusion of the UVABS component allows us to match the high $\mathrm{He} \mathrm{II} / \mathrm{H} \beta$ ratio. UVABS also provides a significantly better fit to the other He II lines and the high-ionization N IV], [Ne IV], and [Ne V] lines (note that He II $\lambda 3204$ is in a noisy region of the spectrum and has a large uncertainty) ${ }^{3}$. Even with the inclusion of UVABS, our model underpredicts the $[\mathrm{Ne} \mathrm{V}] / \mathrm{H} \beta$ ratio somewhat. This cannot be corrected with a harder SED because it would lead to an overprediction of $\mathrm{He} \mathrm{II} / \mathrm{H} \beta$, as discussed earlier in this section. One plausible explanation is that the ionizing continuum has been modified by optically thin gas closer to the nucleus, which has absorbed some of the radiation near the He II Lyman limit but has no effect at energies $\geq 97 \mathrm{eV}$ (the ionization potential of $\left.\mathrm{Ne}^{+3}\right)$. The result would be that the $[\mathrm{Ne} \mathrm{V}]$ would be enhanced with respect to He II, as demonstrated in Kraemer et al. (2000).

Based on our model predictions, we can constrain the covering factors of the individual components. The extinction-corrected $\mathrm{H} \beta$ luminosity of the central knot is $6.4 \times 10^{39} \mathrm{erg}$ $\mathrm{s}^{-1}$. Based on the relative contribution to $\mathrm{H} \beta$ from each model and the emitted flux of $\mathrm{H} \beta$ at the ionized face (Table 3 ), we derive the following covering fractions: for UVABS, $C_{g}=$ 1.1 ; for OIII, $C_{g}=0.02$; and for DENSE, $C_{g}=0.03$.

The global covering factor of the UVABS component is close to one, which, given our assumptions, is consistent with the derived value of $C_{g}=0.5-1.0$ for intrinsic UV absorption in Seyfert galaxies (Crenshaw et al. 1999). Increasing the column density $N_{H}$ of UVABS would decrease $C_{g}$, but this would decrease the $\mathrm{He} \mathrm{II} / \mathrm{H} \beta$ ratio significantly below the observed value ${ }^{4}$. We conclude that there is strong evidence for a high-ionization, matter-bound component in the nuclear knot of NGC 4151 with a high global covering factor.

\footnotetext{
${ }^{3}$ To explore the possible contribution to He II from a collisionally ionized plasma, we assumed the gas is in rough thermal equilibrium with our emission line components, and derived a density $\mathrm{n}(\mathrm{H})=10^{2.5}$ $\mathrm{cm}^{-3}$. This permits a column density of $\sim 10^{22} \mathrm{~cm}^{-2}$ within the central knot. We then generated a model of collisionally ionized plasma with these initial conditions using Cloudy90. The predicted emitted He II $\lambda 4686$ flux was $4.27 \times 10^{-3} \mathrm{ergs} \mathrm{cm}^{-2} \mathrm{~s}^{-1}$, which is 0.08 the contribution from UVABS, or 0.028 of the total observed He II. Hence, this component, which, of course would contribute less if the temperature were higher, cannot contribute significantly to the observed He II emission.

${ }^{4}$ One component in NGC 4151 ("D+E" in Kraemer et al. 2001b) shows evidence for partial covering in the line-of sight, which indicates partial global covering. However, this component is much closer to the source and has a much higher column density than UVABS; there are other UV absorption components in NGC 4151 that may fully cover the continuum source.
} 


\section{Discussion}

We have shown that the bright central knots of emission in the NLRs of most Seyfert galaxies are likely sources of intrinsic absorption lines that we detect in their UV spectra, particularly absorbers that are outflowing with radial velocities $v_{r}<-300 \mathrm{~km} \mathrm{~s}^{-1}$. Our evidence is based on the similarities in the kinematics and locations of the NLR knots and the UV absorbers. More work must be done to test these connections. In particular, more information is needed on the distances of the UV absorbers from their central continuum sources, based on monitoring campaigns and/or detection of metastable lines (see Crenshaw et al. 2003a). We note that intrinsic absorption lines that are within a few hundred $\mathrm{km} \mathrm{s}^{-1}$ of the systemic velocity can arise from either the AGN or host galaxy; the percentage from each is not known and deserves further study.

To establish a more direct link between the emission and absorption, we modeled the narrow emission lines from the resolved central knot in NGC 4151. We found that the line ratios indicate a high-ionization, matter-bound component in the nuclear emission-line knot. Our characterization of this component in the form of "UVABS" yields a high global covering factor, which matches that found for intrinsic UV absorption in Seyfert 1 galaxies $\left(C_{g} \approx 0.5-1\right)$. Given the physical conditions of UVABS, it would be detected as a typical UV absorber if seen projected against the continuum source and BLR. We note that the observed UV spectrum of NGC 4151 shows components of absorption that span a wide range in ionization and column density (Kraemer et al. 2001b), but not a component that specifically matches our UVABS model component for the central emission-line knot. This is likely due to the fact that we are viewing the AGN at a special angle, close to the edge of the emission-line bicone (Crenshaw et al. 2000), which may explain its complex absorption spectrum (Kraemer et al. 2001b). The UVABS component could be "hidden" in the complex of absorption, specifically in the broad component " $\mathrm{D}+\mathrm{E}$ ", or just not present in the line of sight due to a variation in absorption properties with polar angle (with respect to the accretion and/or torus axes). Nevertheless, an important follow-up study would be to model the emission-line spectra of central knots in other Seyfert galaxies, and compare these with their absorption-line properties to test the generality of our results.

A number of dynamical models invoke accretion-disk winds to explain the intrinsic absorption (and broad-line emission) in AGN, using radiation pressure (Murray et al. 1985; Proga et al. 2000) and/or centrifugal acceleration along magnetic field lines (Blandford \& Payne 1982; Bottorff et al. 2000). The absorbers in these models are located close to the BLR, which is only light-days from the central continuum source (presumably the accretion disk around the central supermassive black hole). However, our results suggest that a large fraction, and perhaps a majority, of UV absorbers are located in the inner NLRs 
of Seyfert galaxies, at distances of tens of parsecs from their central continuum sources, and are therefore not directly associated with accretion-disk winds. This does not rule out an origin in the accretion disk, but other origins for the outflowing gas, such as the torus (Krolik \& Kriss 1995) are also possible. Once again, NGC 4151 appears to be an exceptional case, presumably as a result of our special viewing angle. The broad component "D+E" is at a distance of only $\sim 0.03 \mathrm{pc}$ from the continuum source (Kraemer et al. 2001b), and may be the best candidate for an accretion-disk wind in a Seyfert galaxy. There are also outflowing absorbers at large distances from the nucleus ( $\sim 00$ and $\sim 2100$ pc) in NGC 4151 , which may be associated with emission-line clouds further out in the NLR or in the extended NLR (ENLR). Some of the absorbers in other Seyfert galaxies may also arise in these more distant regions.

Finally, we note that X-ray absorbers, which tend to have higher column densities than UV absorbers and likely dominate the mass outflow rates, may also be associated with the NLR. Spectra at the highest possible resolutions with HST/STIS and the Chandra X-ray Observatory $(C X O)$ indicate that the UV and X-ray absorption in individual Seyfert galaxies cover very similar ranges in radial velocity (Crenshaw et al. 2003a, and references therein). This suggests that the UV and X-ray absorbers may be spatially colocated; for example the UV absorbers could be high-density knots in an X-ray wind (Krolik \& Kriss 1995, 2001). Also, CXO observations of NGC 4151 and NGC 1068 (Ogle et al. 2000, 2003) show extended $\mathrm{X}$-ray emission-line regions that are colocated with their NLRs, which would likely be seen as X-ray absorbers if projected against the continuum source. Further identification of components in both emission and absorption should lead to much tighter constraints on the physical conditions, geometry, and dynamics of the outflowing gas.

Some of the data presented in this paper were obtained from the Multimission Archive at the Space Telescope Science Institute (MAST). STScI is operated by the Association of Universities for Research in Astronomy, Inc., under NASA contract NAS5-26555. This research has made use of NASA's Astrophysics Data System. 


\section{REFERENCES}

Bromage, G.E., et al. 1985, MNRAS, 215, 1

Cecil, C., Dopita, M.A., Groves, B., Wilson, A.S., Ferruit, P., Pécontal, E., \& Binette, L. 2002, ApJ, 568, 627

Collinge, M.J., et al. 2001, ApJ, 557, 2

Crenshaw, D.M., Kraemer, S.B., Boggess, A., Maran, S.P., Mushotzky, R.F., \& Wu, C.-C. 1999, ApJ, 516, 750

Crenshaw, D.M., Kraemer, S.B., \& Gabel, J.R. 2004a, in AGN Physics with the Sloan Digital Sky Survey, ed. G.T. Richards \& P.B. Hall (San Francisco: ASP), ASP Conference Series, 311, 235

Crenshaw, D.M., Kraemer, S.B., Gabel, J.R., Schmitt, H.R., Filippenko, A.V., Ho, L.C., Shields, J.C., \& Turner, T.J. 2004b, ApJ, 612, 152

Crenshaw, D.M., Kraemer, S.B., \& George, I.M. 2003a, ARA\&A, 41, 117

Crenshaw D.M., \& Peterson, B.M. 1986, PASP, 98, 185.

Crenshaw, D.M., et al. 2000, AJ, 120, 1731

Crenshaw, D.M., et al. 2002, ApJ, 566, 187.

Crenshaw, D.M., et al. 2003b, ApJ, 594, 116.

Espey, B.R., Kriss, G.A., Krolik, J.H., Zheng, W., Tsvetanov, Z., \& Davidsen, A.F. 1998, ApJ, 500, L13

Ferland, G.J., Korista, K.T., Verner, D.A., Ferguson, J.W., Kingdon, J.B., \& Verner, E.M. 1998, PASP, 110, 761

Gabel, J.R., et al. 2003a, ApJ, 583, 178.

Gabel, J.R., et al. 2003b, ApJ, 595, 120

Gabel, J.R., et al. 2004, ApJ, submitted.

George, I.M., Turner, T.J., Netzer, H., Nandra, K., Mushotzky, R.F., \& Yaqoob, T. 1998, ApJS, 114, 73

Grevesse, N. \& Anders, E. 1989, in Cosmic Abundances of Matter, ed. C.J. Waddington (New York: AIP), 1

Hutchings, J.B., Crenshaw, D.M., Kraemer, S.B., Gabel, J.R., Kaiser, M.E., Weistrop, D., \& Gull, T.R. 2002, AJ, 124, 2543.

Hutchings, J.B., et al. 1998, ApJ, 492, L115 
Kaiser, M.E., et al. 2000, ApJ, 528, 260

Kraemer, S.B. \& Crenshaw, D.M. 2000a, ApJ, 532, 256.

Kraemer, S.B. \& Crenshaw, D.M. 2000b, ApJ, 544, 763

Kraemer, S.B., Crenshaw, D.M., \& Gabel, J.R. 2001a, ApJ, 557, 30.

Kraemer, S.B., Crenshaw, D.M., George, I.M., Netzer, H., Turner, T.J., \& Gabel, J.R. 2002, ApJ, 577, 98.

Kraemer, S.B., Crenshaw, D.M., Hutchings, J.B., Danks, A.C., Gull, T.R., Kaiser, M.E., Nelson, C.H., \& Weistrop, D. 2001b, ApJ, 551, 671.

Kraemer, S.B., Crenshaw, D.M., Hutchings, J.B., Gull, T.R., Kaiser, M.E., Nelson, C.H., \& Weistrop, D. 2000, ApJ, 531, 278.

Kraemer, S.B., Crenshaw, D.M., Yaqoob, T., McKernan, B., Gable, J.R., George, I.M., Turner, \& Dunn, J.P. 2003, ApJ, 582, 125.

Kriss, G.A. 2002, in Mass Outflow in Active Galactic Nuclei: New Perspectives, ed. D.M. Crenshaw, S.B. Kraemer, \& I.M. George (San Francisco: ASP), ASP Conference Series, 255, 69

Kriss, G.A., Blustin, A., Branduardi-Raymont, G., Green, R.F., Hutchings, J., \& Kaiser, M.E. 2003, A\&A, 403, 473

Kriss, G.A., Krolik, J., Grimes, J., Tzvetanov, Z., Espey, B., Zheng, W., \& Davidsen, A. 1997, in Emission Lines in Active Galaxies: New Methods and Techniques, ed. B.M. Peterson, F.-Z. Cheng, \& A.S. Wilson, (San Francisco: ASP), ASP Conference Series, 113,453

Kriss, G.A., et al. 2000, ApJ, 538, L17

Krolik, J.H. \& Kriss, G.A. 1995, ApJ, 447, 512

Krolik, J.H. \& Kriss, G.A. 2001, ApJ, 561, 684

Mathur, S., Elvis, M., \& Wilkes, B. 1995, ApJ, 452, 230

Nelson, C.H., Weistrop, D., Hutchings, J.B., Crenshaw, D.M., Gull, T.R., Kaiser, M.E., Kraemer, S.B., \& Lindler, D. 2000, ApJ, 531, 257.

Netzer, H., Chelouche, D., George, I.M., Turner, T.J., Crenshaw, D.M., Kraemer, S.B., \& Nandra, K. 2002, ApJ, 571, 256

Netzer, H. \& Laor, A. 1993, ApJ, 404, L51

Netzer, H., et al. 2003, ApJ, 599, 933.

Ogle, P.M., et al. 2000, A\&A, 402, 849 
Ogle, P.M., et al. 2003, ApJ, 545, L81

Oliva, E. 1997, in ASP Conference Ser. 113, Emission Lines in Active Galaxies: New Methods and Techniques, ed. B.M Peterson, F.-Z. Cheng, \& A.S. Wilson (San Francisco: ASP), 288

Osterbrock, D.E. 1989, Astrophysics of Gaseous Nebulae and Active Galactic Nuclei (University Science Books: Mill Valley)

Reynolds, C.S. 1997, MNRAS, 286, 513

Ruiz, J.R., Crenshaw, D.M., Kraemer, S.B., Bower, G.A., Gull, T.R., Hutchings, J.B., Kaiser, M.E. and Weistrop, D. 2005, AJ, in press (astro-ph/0409754)

Savage, B.D., \& Mathis, J.S. 1979, ARAA, 17, 73

Schlegel, D.J., Finkbeiner, D.P., \& Davis, M. 1998, ApJ, 500, 525

Schmitt, H.R., Donley, J.L., Antonucci, R.R.J., Hutchings, J.B., \& Kinney, A.L. 2003a, ApJS, 148, 327

Schmitt, H.R., Donley, J.L., Antonucci, R.R.J., Hutchings, J.B., Kinney, A.L., \& Pringle, J.E. 2003b, ApJ, 597, 768

Scott, J.E., et al. 2004, ApJS, 152, 1

Weymann, R.J., Morris, S.K., Gray, M.E., \& Hutchings, J.B. 1997, ApJ, 483, 717 
Fig. 1.- Full-width at half-maximum (FWHM) vs. radial-velocity centroid for individual components of intrinsic absorption in Seyfert 1 galaxies.

Fig. 2.- STIS UV/optical spectrum of the central emission-line knot in NGC 4151, obtained when the continuum and broad-line emission were in a low state. Narrow emission lines that we detected and identified are labeled.

Fig. 3.- Medium-dispersion high-state spectrum of the central emission-line knot in NGC 4151, in the region around $\mathrm{H} \beta$ and [O III] $\lambda \lambda 4959,5007$. 
Table 1. Seyfert Galaxies with STIS Slitless Spectra

\begin{tabular}{lcccccc}
\hline \hline Object & $\begin{array}{c}\text { Seyfert } \\
\text { Type }\end{array}$ & Redshift & $\begin{array}{c}\text { Spatial } \\
\text { HWHM } \\
\left({ }^{\prime \prime}\right)\end{array}$ & $\begin{array}{c}\text { Spatial } \\
\text { HWHM } \\
(\mathrm{pc})^{a}\end{array}$ & $\begin{array}{c}\text { Velocity } \\
\text { HWHM } \\
\left(\mathrm{km} \mathrm{s}^{-1}\right)\end{array}$ & $\begin{array}{c}\text { Velocity } \\
\text { HWZI } \\
\left(\mathrm{km} \mathrm{s}^{-1}\right)\end{array}$ \\
\hline Mrk 573 & 2 & 0.0172 & 0.20 & 66 & 100 & 340 \\
Mrk 620 & 2 & 0.0061 & 0.14 & 17 & 90 & 310 \\
NGC 1386 & 2 & 0.0031 & 0.24 & 14 & 440 & 860 \\
NGC 4151 & 1 & 0.0033 & 0.11 & 7 & 150 & 1060 \\
NGC 3081 & 2 & 0.0079 & $\leq 0.07$ & $\leq 11$ & 100 & 500 \\
NGC 3516 & 1 & 0.0083 & $\leq 0.07$ & $\leq 11$ & 110 & 670 \\
NGC 5506 & 1.9 & 0.0061 & $\leq 0.07$ & $\leq 8$ & 60 & 800 \\
NGC 5643 & 2 & 0.0040 & 0.16 & 12 & 110 & 680 \\
NGC 5728 & 2 & 0.0093 & 0.20 & 36 & 140 & 730 \\
NGC 7212 & 2 & 0.0267 & 0.13 & 67 & 260 & 1010 \\
\hline
\end{tabular}

${ }^{\mathrm{a}}$ For $\mathrm{H}_{0}=75 \mathrm{~km} \mathrm{~s}^{-1} \mathrm{Mpc}^{-1}$

Table 2. STIS Spectra of the Nuclear Knot in NGC 4151

\begin{tabular}{ccrccrr}
\hline \hline $\begin{array}{c}\text { Date } \\
(\mathrm{UT})\end{array}$ & Detector & Grating & $\begin{array}{c}\text { Range } \\
(\AA)\end{array}$ & $\begin{array}{c}\text { Aperture } \\
\left({ }^{\prime \prime}\right)\end{array}$ & $\begin{array}{c}\text { P.A. }^{a} \\
\left({ }^{\circ}\right)\end{array}$ & $\begin{array}{c}\text { Exposure } \\
(\mathrm{s})\end{array}$ \\
\hline 2000 May 28 & MAMA & G140L & $1150-1717$ & $52 \times 0.1$ & 83 & 2220 \\
2000 May 24 & CCD & G230LB & $1672-3077$ & $52 \times 0.1$ & 83 & 2825 \\
2000 May 24 & CCD & G430L & $2906-5715$ & $52 \times 0.1$ & 83 & 780 \\
2000 May 24 & CCD & G750L & $5277-10269$ & $52 \times 0.1$ & 83 & 780 \\
1997 July 15 & CCD & G430M & $4817-5098$ & $52 \times 52$ & 45 & 2139 \\
1997 July 15 & CCD & G750M & $6294-6862$ & $52 \times 52$ & 45 & 1860 \\
\hline
\end{tabular}

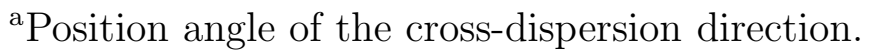


Table 3. NGC 4151 Nuclear Knot- Narrow Emission-Line Ratios (Relative to $\mathrm{H} \beta^{a}$ )

\begin{tabular}{|c|c|c|c|c|c|c|}
\hline \multirow[b]{2}{*}{ Emission Line } & \multicolumn{2}{|c|}{ Observations } & \multicolumn{4}{|c|}{ Models } \\
\hline & Observed & Dereddened $^{b}$ & Composite $^{c}$ & $\mathrm{UVABS}^{d, e}$ & $\mathrm{OIII}^{f}$ & DENSE $^{g}$ \\
\hline N IV] $\lambda 1486$ & $0.75 \pm 0.14$ & $0.81 \pm 0.20$ & 0.67 & 1.82 & 0.32 & 0.65 \\
\hline He II $\lambda 1640$ & $2.00 \pm 0.32$ & $2.15 \pm 0.46$ & 2.22 & 6.88 & 1.58 & 1.60 \\
\hline O III] $\lambda 1663$ & $0.50 \pm 0.08$ & $0.54 \pm 0.12$ & 1.05 & 0.17 & 0.57 & 1.63 \\
\hline $\mathrm{N}$ III] $\lambda 1750$ & $0.44 \pm 0.07$ & $0.47 \pm 0.10$ & 0.45 & 0.15 & 0.26 & 0.67 \\
\hline C III] $\lambda 1909$ & $3.12 \pm 0.46$ & $3.39 \pm 0.72$ & 4.77 & 1.27 & 3.08 & 6.90 \\
\hline O III]/C II] $\lambda 2324$ & $0.38 \pm 0.08$ & $0.41 \pm 0.11$ & 0.58 & - & 0.34 & 0.90 \\
\hline$[\mathrm{Ne}$ IV] $\lambda 2423$ & $0.44 \pm 0.08$ & $0.47 \pm 0.10$ & 0.35 & 1.34 & 0.39 & 0.09 \\
\hline O III $\lambda 3133$ & $0.70 \pm 0.11$ & $0.72 \pm 0.12$ & & & & \\
\hline He II $\lambda 3204$ & $0.18 \pm 0.05$ & $0.19 \pm 0.05$ & 0.12 & 0.35 & 0.08 & 0.09 \\
\hline$[\mathrm{Ne} V] \lambda 3346$ & $0.55 \pm 0.10$ & $0.56 \pm 0.10$ & 0.38 & 1.94 & 0.15 & 0.19 \\
\hline$[\mathrm{Ne} \mathrm{V}] \lambda 3424$ & $1.66 \pm 0.23$ & $1.69 \pm 0.24$ & 1.03 & 5.23 & 0.41 & 0.50 \\
\hline$[\mathrm{Fe}$ VII $] \lambda 3588$ & $0.21 \pm 0.04$ & $0.21 \pm 0.04$ & & & & \\
\hline$[\mathrm{O}$ II] $\lambda 3727$ & $0.33 \pm 0.09$ & $0.34 \pm 0.09$ & 0.11 & - & 0.25 & 0.02 \\
\hline$[\mathrm{Fe}$ VII $] \lambda 3760$ & $0.33 \pm 0.07$ & $0.33 \pm 0.07$ & & & & \\
\hline$[\mathrm{Ne}$ III $] \lambda 3869$ & $1.92 \pm 0.27$ & $1.94 \pm 0.27$ & 1.92 & 0.07 & 1.69 & 2.54 \\
\hline$[\mathrm{Ne}$ III] $\lambda 3967, \mathrm{H} \epsilon$ & $0.56 \pm 0.09$ & $0.56 \pm 0.09$ & & & & \\
\hline [S II $] \lambda 4072$ & $0.33 \pm 0.08$ & $0.33 \pm 0.08$ & 0.26 & - & 0.13 & 0.32 \\
\hline $\mathrm{H} \delta \lambda 4100$ & $0.43 \pm 0.09$ & $0.44 \pm 0.09$ & 0.26 & 0.26 & 0.26 & 0.26 \\
\hline $\mathrm{H} \gamma \lambda 4340$ & $0.75 \pm 0.15$ & $0.75 \pm 0.16$ & 0.47 & 0.47 & 0.47 & 0.47 \\
\hline [O III] $\lambda 4363$ & $0.87 \pm 0.16$ & $0.88 \pm 0.16$ & 0.92 & 0.05 & 0.35 & 1.56 \\
\hline He II $\lambda 4686$ & $0.30 \pm 0.05$ & $0.30 \pm 0.05$ & 0.30 & 0.89 & 0.22 & 0.21 \\
\hline $\mathrm{H} \beta \lambda 4861$ & 1.00 & 1.00 & 1.00 & 1.00 & 1.00 & 1.00 \\
\hline [O III] $\lambda 4959$ & $3.64 \pm 0.46$ & $3.63 \pm 0.46$ & 4.53 & 0.46 & 7.21 & 3.48 \\
\hline [O III] $\lambda 5007$ & $12.17 \pm 1.55$ & $12.14 \pm 1.55$ & 13.61 & 1.39 & 21.65 & 10.43 \\
\hline [Fe VII] $\lambda 5159$ & $0.12 \pm 0.02$ & $0.12 \pm 0.02$ & & & & \\
\hline$[\mathrm{Fe} \mathrm{VI}] \lambda 5176$ & $0.14 \pm 0.02$ & $0.14 \pm 0.02$ & & & & \\
\hline [Fe VII] $\lambda 5721$ & $0.24 \pm 0.04$ & $0.24 \pm 0.04$ & & & & \\
\hline He I $\lambda 5876$ & $0.09 \pm 0.03$ & $0.09 \pm 0.03$ & 0.11 & - & 0.11 & 0.13 \\
\hline [Fe VII] $\lambda 6087$ & $0.42 \pm 0.06$ & $0.41 \pm 0.07$ & 0.15 & 0.29 & 0.12 & 0.14 \\
\hline$[\mathrm{O}$ I] $\lambda 6300$ & $0.63 \pm 0.10$ & $0.62 \pm 0.11$ & 0.55 & - & 0.39 & 0.80 \\
\hline$[\mathrm{O} \mathrm{I}],[\mathrm{Fe} \mathrm{X}] \lambda 6374$ & $0.33 \pm 0.06$ & $0.32 \pm 0.06$ & 0.25 & 0.50 & 0.13 & 0.27 \\
\hline$[\mathrm{N}$ II $] \lambda 6548$ & $0.50 \pm 0.14$ & $0.49 \pm 0.14$ & 0.25 & - & 0.37 & 0.23 \\
\hline $\mathrm{H} \alpha \lambda 6563$ & $3.50 \pm 0.66$ & $3.42 \pm 0.67$ & 2.91 & 2.71 & 2.89 & 2.98 \\
\hline [N II] $\lambda 6584$ & $1.50 \pm 0.42$ & $1.47 \pm 0.42$ & 0.76 & - & 1.11 & 0.68 \\
\hline$[\mathrm{S} \mathrm{II}] \lambda 6716$ & $0.08 \pm 0.01$ & $0.07 \pm 0.01$ & 0.04 & - & 0.09 & 0.02 \\
\hline$[\mathrm{S} \mathrm{II}] \lambda 6731$ & $0.18 \pm 0.02$ & $0.18 \pm 0.02$ & 0.10 & - & 0.19 & 0.05 \\
\hline$[\mathrm{Ar} \mathrm{V}] \lambda 7005$ & $0.07 \pm 0.01$ & $0.06 \pm 0.01$ & 0.03 & 0.01 & 0.03 & 0.04 \\
\hline
\end{tabular}


Table 3-Continued

\begin{tabular}{|c|c|c|c|c|c|c|}
\hline \multirow[b]{2}{*}{ Emission Line } & \multicolumn{2}{|c|}{ Observations } & \multicolumn{4}{|c|}{ Models } \\
\hline & Observed & Dereddened $^{b}$ & Composite $^{c}$ & $\mathrm{UVABS}^{d, e}$ & $\mathrm{OIII}^{f}$ & DENSE $^{g}$ \\
\hline He I $\lambda 7065$ & $0.10 \pm 0.01$ & $0.09 \pm 0.01$ & 0.08 & - & 0.08 & 0.09 \\
\hline$[$ Ar III] $\lambda 7136$ & $0.24 \pm 0.03$ & $0.23 \pm 0.04$ & 0.23 & - & 0.25 & 0.26 \\
\hline$[\mathrm{O}$ II] $\lambda 7325$ & $0.29 \pm 0.04$ & $0.28 \pm 0.05$ & 0.22 & - & 0.20 & 0.28 \\
\hline [ Ar III $] \lambda 7751$ & $0.06 \pm 0.01$ & $0.06 \pm 0.01$ & 0.05 & - & 0.06 & 0.06 \\
\hline$[\mathrm{Fe}$ XI] $\lambda 7892$ & $0.10 \pm 0.02$ & $0.10 \pm 0.03$ & 0.01 & 0.08 & - & - \\
\hline$[\mathrm{S}$ III $] \lambda 9069$ & $0.32 \pm 0.05$ & $0.31 \pm 0.05$ & 0.35 & - & 0.55 & 0.29 \\
\hline [S III] $\lambda 9532$ & $0.91 \pm 0.15$ & $0.88 \pm 0.16$ & 0.88 & 0.01 & 1.37 & 0.71 \\
\hline
\end{tabular}

${ }^{a}$ Observed flux of $\mathrm{H} \beta$ from entire knot (G430M spectrum) $=3.0( \pm 0.4) \times 10^{-13} \mathrm{ergs} \mathrm{s}^{-1}$ $\mathrm{cm}^{-2}$

${ }^{\mathrm{b}}$ Used $\mathrm{E}_{B-V}=0.02$ and standard Galactic reddening curve (Savage \& Mathis 1979.

${ }^{\mathrm{c}}$ Weighted according to the fraction of $\mathrm{H} \beta$ from each model component: 0.12 (UVABS), 0.38 (OIII), 0.50 (DENSE).

$$
\begin{aligned}
& { }^{\mathrm{d}} U=0.03, n_{H}=10^{4.3} \mathrm{~cm}^{-3}, N_{H}=10^{19.5} \mathrm{~cm}^{-2}, \mathrm{~d}=9.5 \mathrm{pc}, \mathrm{f}_{H \beta}=5.6 \times 10^{-2} \mathrm{ergs} \mathrm{s}^{-1} \\
& \mathrm{~cm}^{-2}, C_{g}=1.1 .
\end{aligned}
$$

${ }^{\mathrm{e}}$ Ionic column densities for UVABS are N(H I $)=4.5 \times 10^{15} \mathrm{~cm}^{-2}, \mathrm{~N}(\mathrm{C}$ IV $)=1.5 \times$ $10^{15} \mathrm{~cm}^{-2}, \mathrm{~N}(\mathrm{~N} \mathrm{~V})=1.3 \times 10^{15} \mathrm{~cm}^{-2}, \mathrm{~N}(\mathrm{O}$ VI $)=5.3 \times 10^{15} \mathrm{~cm}^{-2}$, and $\mathrm{N}(\mathrm{Si} \mathrm{IV})=3.1$ $\times 10^{12} \mathrm{~cm}^{-2}$.

$$
\begin{aligned}
& { }^{\mathrm{f}} U=0.01, n_{H}=10^{4.8} \mathrm{~cm}^{-3} \text {, radiation bound, } \mathrm{d}=9.5 \mathrm{pc}, \mathrm{f}_{H \beta}=8.1 \mathrm{ergs} \mathrm{s}^{-1} \mathrm{~cm}^{-2}, C_{g} \\
& =0.02 \\
& { }^{\mathrm{g}} U=0.01, n_{H}=10^{6.2} \mathrm{~cm}^{-3}, \text { radiation bound, } \mathrm{d}=2.0 \mathrm{pc}, \mathrm{f}_{H \beta}=2.1 \times 10^{2} \mathrm{ergs} \mathrm{s}^{-1} \mathrm{~cm}^{-2}, \\
& C_{g}=0.03
\end{aligned}
$$




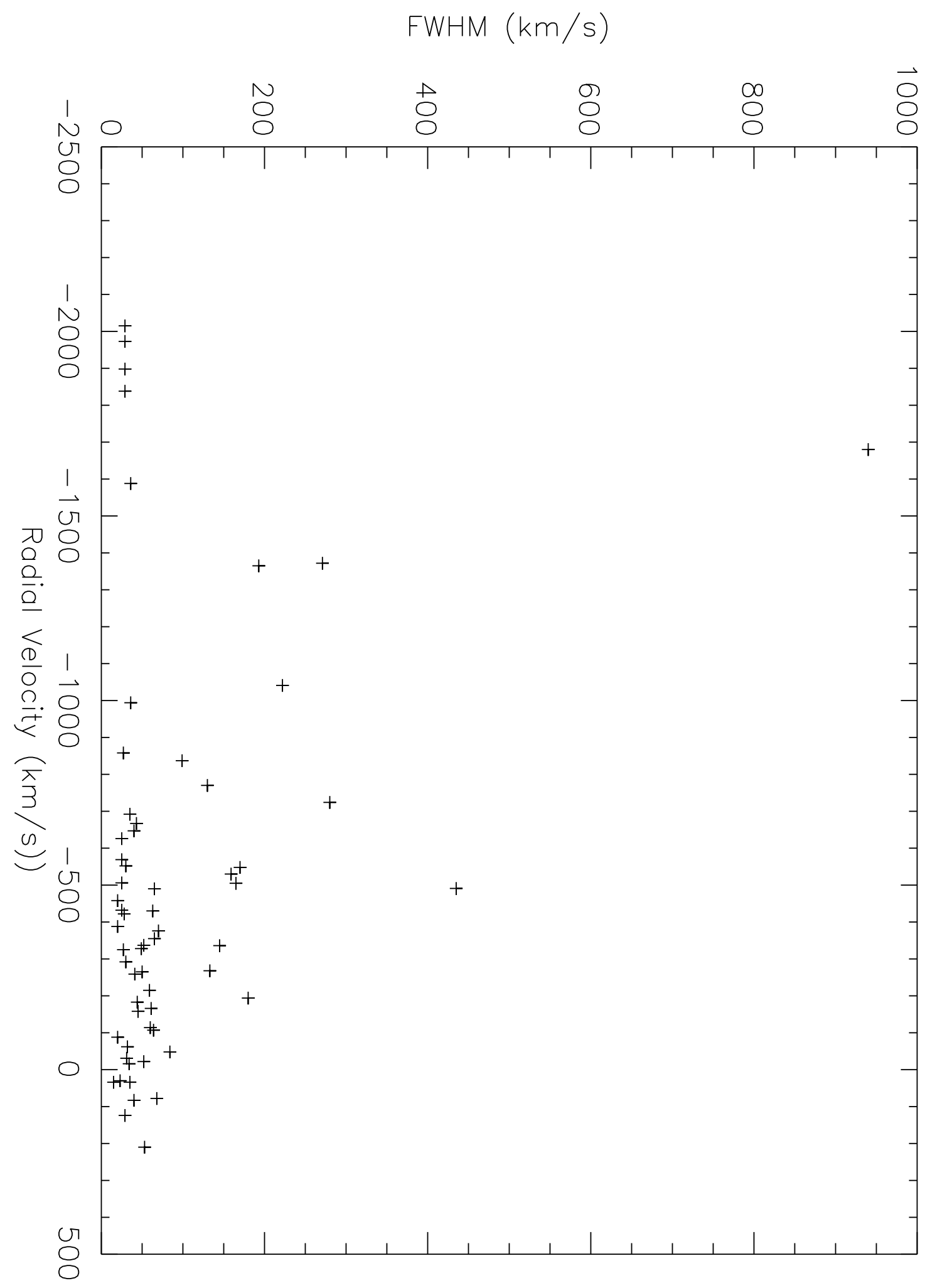

Fig. 1. 

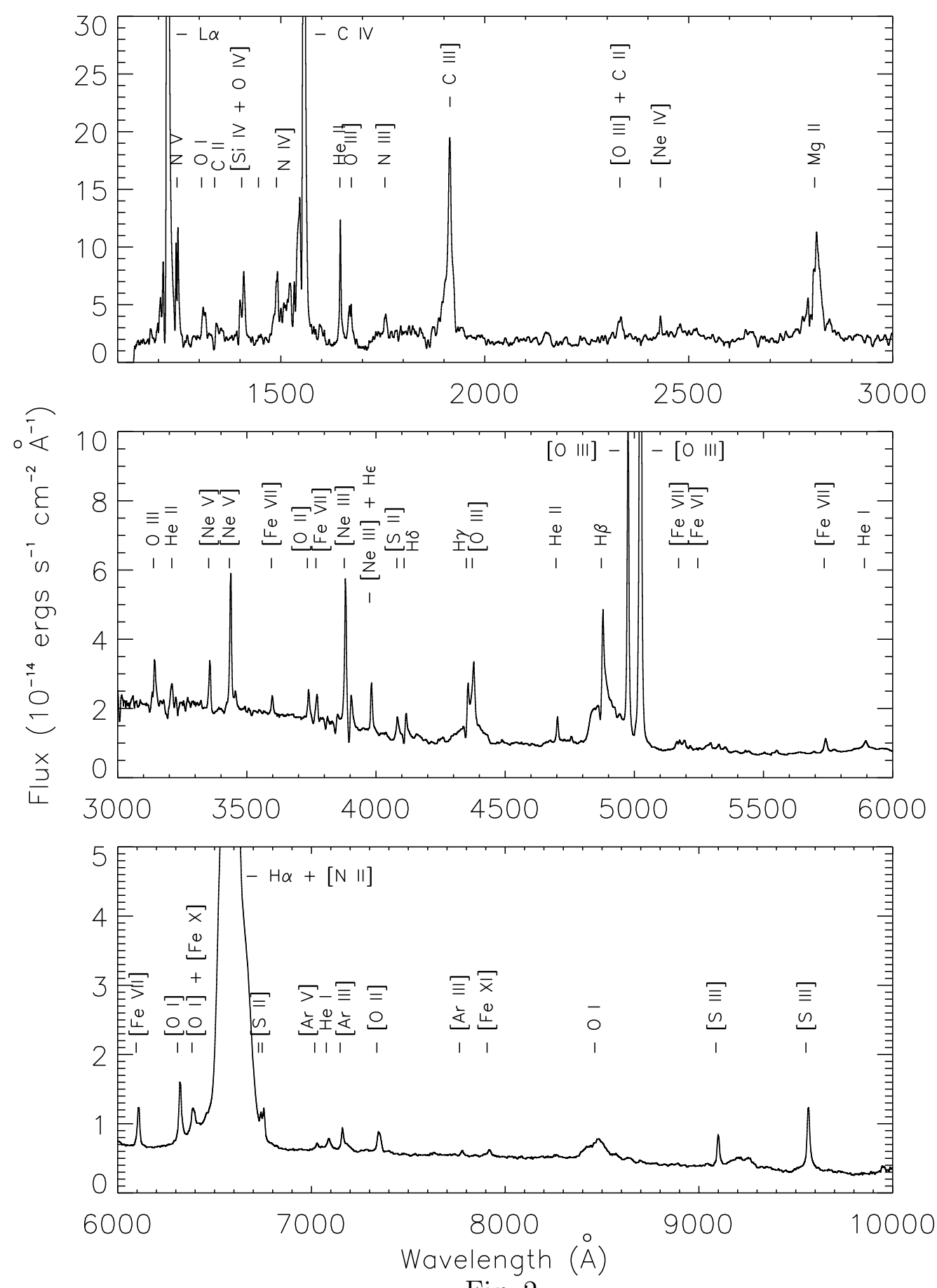

Fig. 2. 


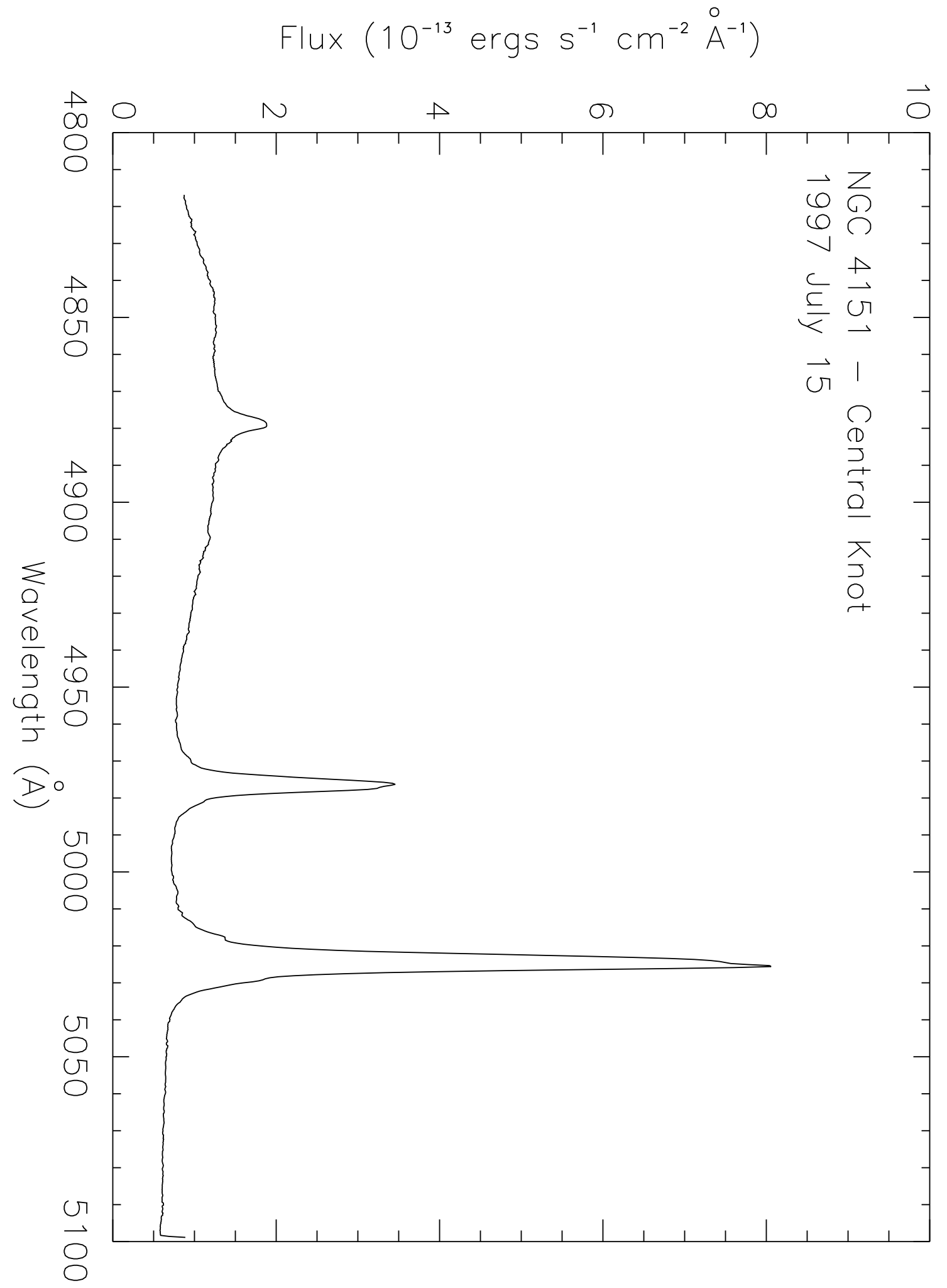

Fig. 3. 